\title{
Efectos de insecticidas botánicos comerciales en Tamarixia radiata, un ectoparasitoide de Diaphorina citri
}

\section{Effects of commercial botanical insecticides in Tamarixia radiata, an ectoparasitoid of Diaphorina citri}

\author{
Raúl José Monsreal-Ceballos ${ }^{1}$, Esaú Ruíz-Sánchez ${ }^{1}$, Maricarmen Sánchez Borja ${ }^{2}$, Horacio Salomón \\ Ballina-Gómez ${ }^{1 *}$, Alejandra González-Moreno ${ }^{1}$, Arturo Reyes-Ramirez ${ }^{1}$ \\ ${ }^{1}$ Departamento de Posgrado e Investigación, Tecnológico Nacional de México, Instituto Tecnológico de Conkal. Avenida \\ Tecnológico s/n, CP. 97345. Mérida, Yucatán. México. \\ ${ }^{2}$ Comité Estatal de Sanidad Vegetal. Calle 19 No. 443 entre 26 y 28, CP. 97288. Mérida, Yucatán, México. \\ *Autor de correspondencia: horacio.ballina @itconkal.edu.mx
}

Nota científica recibida: 05 de octubre de 2016 aceptada: 17 de marzo de 2017

RESUMEN. Se evaluó el efecto de tres insecticidas botánicos comerciales sobre la mortalidad por residualidad e ingestión en Tamarixia radiata. Se observó que el Biodie y NeeemPHC produjeron mortalidad del 12.7 y $12.3 \%$, por efecto de residual, respectivamente; y del 33.3 y $28.7 \%$ mortalidad por ingestión. El tiempo también incrementó de forma significativa la mortalidad, aunque sólo por ingestión y hasta las 48 y $72 \mathrm{~h}$ con Biodie, y el mismo período por el NeeemPHC. El CinnAcar fue poco tóxico por ingestión y ninguno de los insecticidas botánicos tuvieron efectos significativos en la emergencia de adultos. Los tres productos evaluados no interfieren en el control biológico.

Palabras clave: Control biológico, efectos residuales, ingestión, mortalidad, parasitoides

ABSTRACT. The effect of three commercial botanical insecticides on the mortality by residues and ingestion of Tamarixia radiata was evaluated. Biodie and NeemPHC had 12.7 and $12.3 \%$ residue mortality effect and 33.3 and $28.7 \%$ mortality by ingestion, respectively. Mortality also increased significantly as time went by, although only with regard to ingestion, and up to 48 and $72 \mathrm{~h}$, for both Biodie and NeemPHC. CinnAcar did not have a major toxic effect when ingested and none of the botanical insecticides had significant effects on the appearance of adults. The three products under evaluation do not interfere with biological control.

Key words: Biological control, residual effects, ingest, mortality, parasitoids

\section{INTRODUCCIÓN}

Diaphorina citri Kuwayama (Hemiptera: Psilidae) es uno de los principales obstáculos para la producción citrícola a nivel mundial, ya que causa daños directos por alimentación de los estados inmaduros y adultos, además es vector de Candidatus Liberibacter asiaticum, organismo causante de la enfermedad denominada Huanglongbing (HLB) o enverdecimiento de los cítricos (Bové 2006). Una de las principales estrategias adoptadas a nivel mundial para la regulación de $D$. citri es el control biológico clásico con el idiobionte ectoparasitoide Tamarixia radiata Waterson (Hymenoptera: Eulophidae), con evidente éxito en las Islas Reunión, Guadalupe y Puerto Rico (Étienne et al. 2001, Pluke et al. 2008). Aunque también se ha encontrado que, en ciertas zonas, las tasas de parasitismo son bajas y esporádicas (Qureshi et al. 2009). En este sentido, los insecticidas botánicos se han propuesto como productos inocuos para enemigos naturales, ya que poseen menor residualidad que los productos sintéticos (Montes-Molina et al. 2008). No obstante, el conocimiento del efecto de los insecticidas botánicos en este himenóptero es muy escaso, por lo que el presente estudio tuvo como objetivo evaluar los efectos letales y subletales de insecticidas botánicos comerciales sobre el control de $T$. radiata bajo 
condiciones de laboratorio.

\section{MATERIALES Y MÉTODOS}

El trabajo se realizó en las instalaciones del Laboratorio Regional de Producción Masiva de Tamarixia radiata del Sureste, perteneciente al Centro Nacional de Referencia de Control Biológico convenio SENASICA-CESVY, ubicado en el estado de Yucatán, México.

La cría de $D$. citri se estableció bajo condiciones de invernadero infestando tres lotes de 12 plantas de Murraya paniculata, hospedera natural de D. citri. Cada lote de plantas se introdujo en jaulas entomológicas cubiertas con malla antiáfidos de 70 $\times 70 \times 70 \mathrm{~cm}$, con plantas de M. Paniculata con altura aproximada de $50 \mathrm{~cm}$ con dos brotes. Para la infestación se utilizaron 100 psílidos adultos, que tenían seis días libres de parasitación. Luego se introdujeron 140 individuos adultos de $T$. radiata por lote de plantas, después de 9 a $10 \mathrm{~d}$, las plantas con ninfas parasitadas se trasladaron al laboratorio y se introdujeron en una jaula entomológica, para observar la emergencia de adultos que se usaron en los bioensayos de mortalidad y emergencia. Los parasitoides adultos se manipularon con un aspirador manual en todos los bioensayos. El material biológico durante el bioensayo se mantuvo a temperatura de $25 \pm 5{ }^{\circ} \mathrm{C}$ con humedad relativa de 60 $\pm 10 \%$, libre del contacto con pesticidas.

$\mathrm{Se}$ usaron los insecticidas botánicos CinnAcar ${ }^{\circledR}$, Biodie ${ }^{\circledR}$ y PHC Neem $^{\circledR}$, recomendados para el control de psílidos. Para asegurar el máximo efecto potencial sobre los parasitoides, para el CinnAcar ${ }^{\circledR}$ y el Biodie ${ }^{\circledR}$ se usó la dosis más alta recomendada por el fabricante; mientras que para el PHC Neem ${ }^{\circledR}$ se usó la dosis intermedia. El cálculo de la concentración de producto por volumen de agua se realizó tomando como base $400 \mathrm{~L} \mathrm{ha}^{-1}$ (Tabla 1). Mientras que el tratamiento testigo consistió en la aplicación de agua destilada. Todas las dosis de los tres tratamientos se prepararon al momento de la aplicación. Para los bioensayos por contacto residual y por ingestión, se utilizaron frascos de plástico de $100 \mathrm{~mL}$, con un orificio de 2.5 $\mathrm{cm}$ de diámetro en la parte superior de la tapa, cubierto con tela de organza para permitir la entrada de oxígeno.

En los bioensayos por contacto residual, el interior y la tapa de cada frasco se pulverizaron con $2 \mathrm{~mL}$ de cada insecticida botánico o agua destilada (testigo), para luego dejar secar por tres horas a temperatura ambiente. Una vez secos, se introdujeron 20 individuos adultos sin distinción de sexo de 24 a $72 \mathrm{~h}$ de edad por frasco. En los bioensayos los parasitoides se alimentaron con $6.6 \mathrm{~mL}$ de miel de abeja, que se aplicó sobre una tira de papel de 2.5 $\times 8.5 \mathrm{~cm}$

En los bioensayos por ingestión, se usaron 20 adultos por frasco de $T$. radiata sin distinción de sexo con edad de 24 a $72 \mathrm{~h}$, los cuales se alimentaron con una solución de miel y solución insecticida en proporciones de $1: 1$; que se colocó sobre tiras de papel de $2.5 \times 8.5 \mathrm{~cm}$. Cada tratamiento tuvo cinco repeticiones (frasco) con 20 individuos. En los bioensayos, se registró el porcentaje de mortalidad de adultos a las 24,48 y $72 \mathrm{~h}$. Los individuos se consideraron muertos si fueron incapaces de mantener una posición vertical o si no presentaron movimiento al ser estimulados con un pincel y al agitar de forma leve el frasco, cada individuo se observó por al menos $30 \mathrm{~s}$ (Iannacone y Lamas 2003).

Para los bioensayos, se utilizaron plantas con ninfas parasitadas con signos de momificación, hilos de seda alrededor de la ninfa y presencia del meconio, para los estados de prepupa-pupa de los parasitoides (Chien et al. 1991). Cada planta se limpió con una pinza entomológica y un pincel, de manera que al final cada planta de $M$. paniculata tuviera 40 ninfas momificadas. Las plantas se asperjaron homogéneamente con los insecticidas botánicos o agua destilada hasta el punto de escurrimiento, posteriormente se dejaron secar por $15 \mathrm{~min}$ a temperatura ambiente, una vez secas se introdujeron en cubos de acrílico de $29.5 \times 29.5 \times 60 \mathrm{~cm}$, con ventanas cubiertas con malla antiafidos. Durante $15 \mathrm{~d}$ se registró el porcentaje de adultos emergidos, los cuales se retiraron con un aspirador manual. Los resultados se confirmaron al final del biensayo observando de forma directa los brotes experimen- 
Tabla 1. Insecticidas botánicos y concentraciones utilizadas en este estudio.

\begin{tabular}{|c|c|c|c|}
\hline Insecticida & Ingrediente activo & 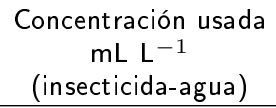 & $\begin{array}{l}\text { Dosis producto } \\
\quad\left(\mathrm{L} \mathrm{ha}^{-1}\right)\end{array}$ \\
\hline CinnAcar ${ }^{\circledR}$ & $\begin{array}{l}\text { Cinnamaldehído y } \\
\text { ácido cinámico } 15 \%\end{array}$ & 7.5 & 1,2 y 3 \\
\hline Biodie ${ }^{\circledR}$ & $\begin{array}{l}\text { Argemonina, Berberina, } \\
\text { Ricininay alfa-tertienil } 12 \%\end{array}$ & 5 & $1,1.5$ y 2 \\
\hline $\begin{array}{l}\text { PHC }^{\circledR} \\
\text { NeeeM }^{\circledR}\end{array}$ & Azadiractina $3 \%$ & 7 & 2 y 4 \\
\hline
\end{tabular}

tales con un microscopio estereoscopio (Leica Microsystems Alemania, modelo MZ6, Zoom 6.3:1), para cuantificar el número de momias con orificio de emergencia. Para cada tratamiento se tuvieron cinco repeticiones de 40 momias cada una.

Los porcentajes de mortalidad y de emergencia se transformaron con la raíz del arcoseno y se analizaron con modelos lineales generalizados (MLG) (McCullagh y Nelder 1989), bajo una estructura de medidas repetidas; con el factor insecticidas botánicos como inter-sujetos, con cuatro niveles, y un factor tiempo como intra-sujetos con tres niveles. Para identificar diferencias entre los niveles del factor insecticida y entre los tres niveles del factor intra-sujetos se utilizaron comparaciones pareadas de Bonferroni. Los análisis se realizaron con el paquete estadístico SPSS 19 Statistics para Windows.

\section{RESULTADOS Y DISCUSIÓN}

En el contacto residual, el efecto causado por el Biodie y el Neeem PHC fue mayor al testigo, con un 12.7 y $12.3 \%$ de mortalidad, respectivamente. Esta variable fue diferente entre los diferentes tiempos de exposición, observándose mayor mortalidad a las 72 h (Tabla 2). La interacción entre los factores tiempo $x$ insecticida no fue significativa (Figura 1A). Los bajos niveles de mortalidad de $T$. radiata puede deberse, a la rápida degradación de los insecticidas botánicos por los factores ambientales como la luz, temperatura y humedad (Wiesbrook 2004). Particularmente, el Biodie, tiene componentes como la fitotoxina, alfa-tertienil, que persiste durante cuatro horas bajo la exposición de la luz del sol (Philogène et al. 1985). En relación al
Neem PHC, se tiene registrado como un producto de toxicidad baja en tratamientos residuales sobre Tamarixia triozae (Burcks) (Luna-Cruz et al. 2011). Se ha demostrado que la residualidad de insecticidas botánicos basados en azadiractina, suelen ser de bajo impacto en especies de himenópteros parasitoides (Xu et al. 2004, Kumar et al. 2010, Sidi et al. 2013). En contraste, los resultados muestran que la exposición residual al CinnAcar no fue letal para $T$. radiata, y aunque no se encontraron antecedentes de los efectos residuales de este producto en la mortalidad de parasitoides, los hallazgos coinciden con lo obtenido por Buczkowski et al. (2005), quienes evaluaron un insecticida compuesto de Cinnamaldehído en Monomorium pharaonis (Hymenoptera: Formicide) y encontraron que no se elevó de forma significativa los niveles de mortalidad bajo tratamientos residuales.

En la ingestión, al igual que en los bioensayos de residualidad, la mortalidad de $T$. radiata sólo fue causada por el Biodie y el Neeem PHC, con porcentajes de mortalidad significativamente mayores que los observados en los bioensayos de exposición residual. Lo que coincide con lo reportado por Liu et al. (2012) quienes observaron que el efecto de un insecticida botánico elaborado con Chenopodium ambrosoides causó mayor efecto por ingestión en Tamarixia trioze. La letalidad causada por el Biodie puede deberse a que está compuesto por alcaloides como la argemonina, berberina, ricinina y un tiofeno denominado alfa-tertienil, que aunque no se han demostrado sus efectos sobre parasitoides, se tienen evidencias de daños letales en otros insectos como Apis mellifera (Hymenoptera: Apidae) (de Assis Junior et al. 2011) y Culex quinquefasciatus (Dipete- 
Monsreal-Ceballos et al.

Tabla 2. Efectos por residualidad e ingestión de insecticidas botánicos en la mortalidad de adultos de $T$. radiata.

\begin{tabular}{|c|c|c|c|c|}
\hline \multirow{2}{*}{ Efecto } & \multicolumn{2}{|c|}{ Residualidad } & \multicolumn{2}{|c|}{ Ingestión } \\
\hline & Mortalidad (\%) & $\chi^{2}$ Wald & Mortalidad (\%) & $\chi^{2}$ Wald \\
\hline \multicolumn{5}{|l|}{ Inter-sujetos } \\
\hline Insecticida (I) & & $25.765^{* *}$ & & $101.126 * *$ \\
\hline Testigo & $4.3 \pm 2.7^{a}$ & & $9.7 \pm 2.8^{a}$ & \\
\hline CinnAcar & $4.7 \pm 2.6^{a}$ & & $11.7 \pm 2.9^{a}$ & \\
\hline Biodie & $12.7 \pm 3.1^{b}$ & & $33.3 \pm 4.6^{b}$ & \\
\hline Neeem PHC & $12.3 \pm 3.8^{b}$ & & $28.7 \pm 4.6^{b}$ & \\
\hline \multicolumn{5}{|l|}{ Intra-sujetos } \\
\hline Tiempo(horas) (T) & & $62.465^{* *}$ & & $161.275^{* *}$ \\
\hline 24 & $1.5 \pm 1.7^{a}$ & & $6.3 \pm 2.7^{a}$ & \\
\hline 48 & $6.8 \pm 2.6^{b}$ & & $21.3 \pm 3.5^{b}$ & \\
\hline 72 & $17.3 \pm 3.6^{c}$ & & $35 \pm 4.6^{c}$ & \\
\hline$T \times 1$ & & $11.185 \mathrm{~ns}$ & & $14.130 *$ \\
\hline
\end{tabular}

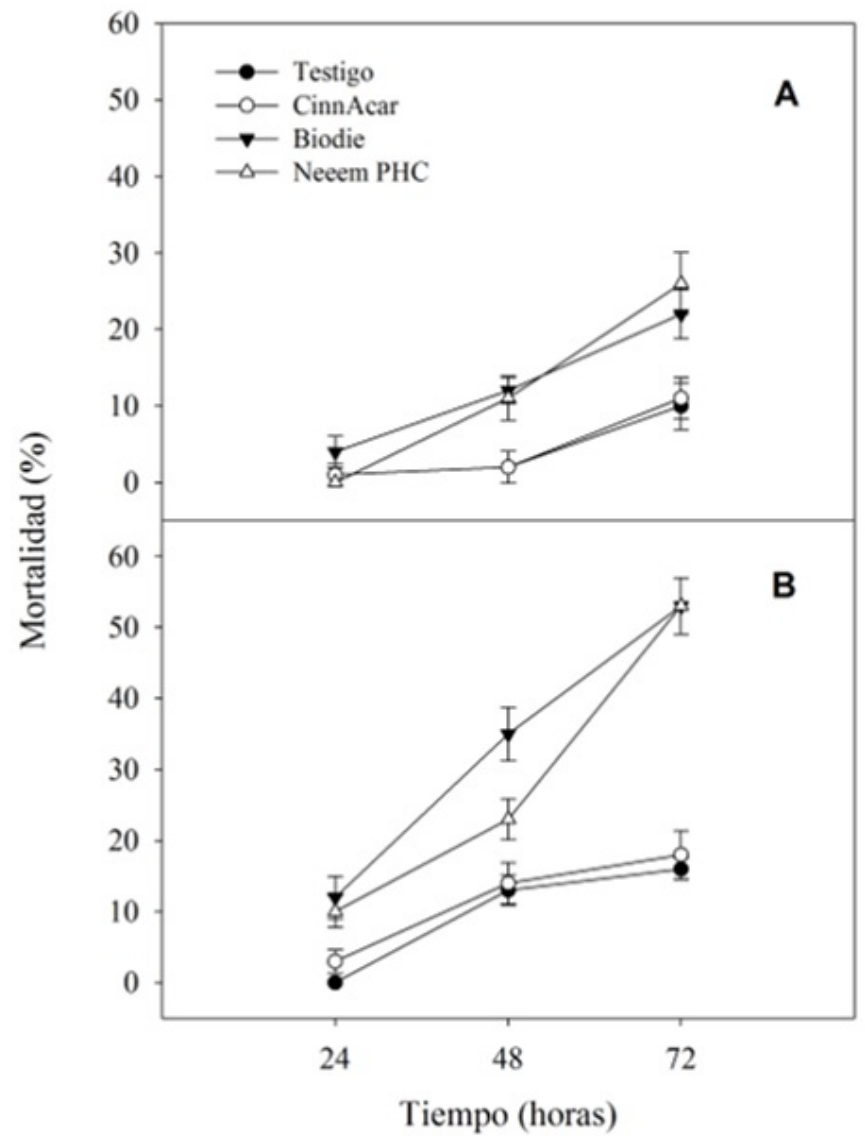

Figura 1. Porcentaje de mortalidad de adultos (medias \pm error) de $T$. radiata expuestos a tratamientos residuales (A) y de ingestión (B) de insecticidas botánicos comerciales, a las 24, 48 y $72 \mathrm{~h}$. 
ra: Culicidae) (Wang et al. 2000). Por otra parte, los efectos significativos de la ingestión del Neeem PHC en la mortalidad de adultos de T. radiata contrastan con estudios que reportan que la azadiractina no tiene efectos en la mortalidad de Himenópteros parasitoides como Cotesia plutellae (Haseeb et al. 2004) y Diadegma insulare (Xu et al. 2004). Estos resultados pueden deberse al tiempo de exposición, ya que en los estudios mencionados se evaluó la mortalidad a las $24 \mathrm{~h}$, mientras que en el presente trabajo la mortalidad se obtuvo a las $72 \mathrm{~h}$ (Tabla 2). La interacción entre el tiempo y el insecticida, indica que el efecto varió con el producto y el tiempo de exposición del parasitoide. A las $24 \mathrm{~h}$ la mortalidad causada por los insecticidas fue similar al testigo, mientras que a las $48 \mathrm{~h}$ el efecto de Biodie fue diferente, mientras que a las $72 \mathrm{~h}$ el efecto del Biodie y el Neem PHC fueron significativamente mayores (Figura 1B). Estos resultados pueden deberse a la capacidad de desintoxicación y excreción de metabolitos entre parasitoides (Morales-Ramos y Rojas 2003). No se observaron efectos significativos por la ingestión del CinnAcar, lo que contrasta con estudios que reportan efectos letales por contacto en coleópteros (Ojimelukwe y Adler 2002) y la actividad antialimentaria en Spodoptera litura (Arivoli y Tennyson 2013). Aunque Huang y Ho (1998) observaron que una concentración de $13 \mathrm{mg} \mathrm{g}^{-1}$ de alimento de cinnamaldehído no causa efectos en el consumo de alimento en Tribolium castenum.

Para el hospedero parasitado, los insecticidas botánicos no disminuyeron los porcentajes de emergencia de adultos de $T$. radiata, siendo su efecto similar al grupo testigo; aunque, el CinnAcar tuvo un efecto marginal (Tabla 3, Bonferroni $p=$ $0.065)$. Pese a que no se encontraron antecedentes de los efectos de este producto sobre parasitoides, se ha observado que el extracto de Cinnamon zeylanicumre puede afectar el desarrollo de insectos. Al respecto Mahdi y Rahman (2008) reportan que el extracto de esta especie redujo la emergencia de adultos de Callosobruchus maculatus. Estos resultados coinciden con otros estudios donde se demuestra el bajo o nulo efecto tópico que tienen los insecticidas botánicos sobre el hospedero parasitado; en general la azadiractina ha mostrado ser de impacto bajo en la emergencia de adultos de Lysiphlebus testaceipes (Tang et al. 2002), Encarsia formosa (Simmonds et al. 2002), E. sofia (Aggarwal y Brar 2006) y T. trioze (Luna-Cruz et al. 2011). Estos resultados contrastan con lo reportado para el Biodie, donde se reporta la supervivencia de las pupas hasta la emergencia de adultos del endoparasitoide (McDougall 1986).

Tabla 3. Efectos de insecticidas botánicos comerciales en la emergencia de adultos por contacto tópico a momias de $D$. citri.

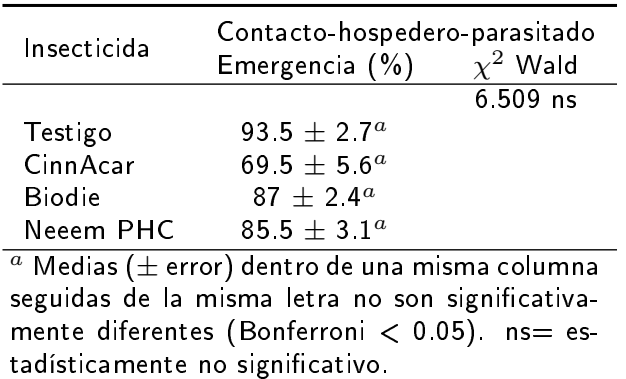

Para Diadegama tenebrans se reporta disminución de la población por efecto de la Berberina y el alfa-tertienil, lo que puede deberse a la metodología en la exposición de los parasitoides, debido a que en el presente trabajo se evaluó el proceso de emergencia bajo un método diferente. El bajo o nulo efecto de los insecticidas botánicos evaluados en la emergencia de adultos de $T$. radiata se puede deber a aspectos biológicos de la especie. Éste himenóptero, pese a ser un ectoparasitoide, tiene un proceso de desarrollo, desde huevo hasta pupa, en la parte ventral de su hospedero; incluso, cuando la larva avanza al estado prepupal asegura la momia a la superfiecie de la planta por medio de hilos de seda (Étienne et al. 2001). Ésta característica le puede ofrecer cierta protección a las aplicaciones tópicas de insecticidas. Al respecto se ha demostrado que parasitoides que se desarrollan dentro del hospedero, tienen cierta protección a las aplicaciones de insecticidas (Ateyyat 2009).

Los tres insecticidas botánicos pueden ser compatibles con $T$. radiata para el manejo integrado de $D$. citri, ya que la exposición residual a los insecticidas no fue letal para adultos del parasitoide. Por 
lo que podrían ser aplicados antes de las liberaciones de $T$. radiata, considerando que este parasitoide se alimenta en estado adulto de ninfas de $D$. citri, por lo que los tratamientos de ingestión adquieren especial importancia. Los resultados sugieren que el CinnAcar puede ser una alternativa sobre el Biodie y el Neem PHC. Las aplicaciones tópicas a momias de
$D$. citri ofrece un acercamiento del efecto que podrían tener los productos cuando son asperjados en los cultivos. Los tres insecticidas pueden ser una alternativa; pero Biodie y Neeem PHC tienen mayores niveles de emergencia de adultos y con ello mayor probabilidad de que se establecieran las poblaciones de $T$. radiata.

\section{LITERATURA CITADA}

Aggarwal N, Brar DS (2006) Effects of different neem preparations in comparison to synthetic insecticides on the whitefly parasitoid Encarsia sophia (Hymenoptera: Aphelinidae) and the predator Chrysoperla carnea (Neuroptera: Chrysopidae) on cotton under laboratory conditions. Journal of Pest Science 79: 201-207.

Arivoli S, Tennyson S (2013) Antifeedant activity, developmental indices and morphogenetic variations of plant extracts against Spodoptera litura (Fab) (Lepidoptera: Noctuidae). Journal of Entomology and Zoology Studies 1: 87-96.

Ateyyat M (2009) Insecticidal and repellent activities of medicinal plant extracts against the sweet potato whitefly, Bemisia tabaci (Homoptera: Aleyrodidae) and its parasitoid Eretmocerus mundus (Hymenopthera: Aphelinidae). Journal of Pest Science 82: 149-154.

Bové JM (2006) Huanglongbing: a destructive, newly-emerging, century-old disease of citrus. Journal of Plant Pathology 88: 7-37.

Buczkowski G, Scharf ME, Ratliff CR, Bennett GW (2005) Efficacy of simulated barrier treatments against laboratory colonies of pharaoh ant. Journal of Economic Entomology 98: 485-492.

Chien CC, Chu YI, Ku SC (1991) Parasitic strategy, morphology and life history of Tamarixia radiata (Hymenoptera; Eulophidae). Chinese Journal of Entomology 11: 264-281.

de Assis Junior EM, dos Santos Fernandes IM, Santos CS, de Mesquita LX, Pereira RA, Maracajá PB, et al. (2011) Toxicity of castor bean (Ricinus communis) pollen to honeybees. Agriculture, Ecosystems and Environment 141: 221-223.

Étienne J, Quilici S, Marival D, Franck A (2001) Biological control of Diaphorina citri (Hemiptera: Psyllidae) in Guadeloupe by imported Tamarixia radiata (hymenoptera: Eulophidae). Fruits 56: 307-315.

Haseeb M, Liu TX, Jones WA (2004) Effects of selected insecticides on Cotesia plutellae, endoparasitoid of Plutella xylostella. BioControl 49: 33-46.

Huang Y, Ho SH (1998) Toxicity and antifeedant activities of cinnamaldehyde against the grain storage insects, Tribolium castaneum (Herbst) and Sitophilus zeamais Motsch. Journal of Stored Products Research 34: 11-17.

lannacone J, Lamas G (2003) Efectos toxicológicos del nim, rotenone y cartap sobre tres micro-avispas parasitoides de plagas agrícolas en el Perú. Boletín Sanidad Vegetal Plagas 29: 123-142.

Kumar P, Singh HP, Poehlings HM (2010) Effects of neem on adults of Eretmocerus warrae (Hym., Aphelinidae), a parasitoid of Bemisia tabaci (Horn., Aleyrodidae) in tropical horticulture systems. Journal of Plant Diseases and Protection 117: 273-277. 
Luna-Cruz A, Lomeli-Flores JR, Rodríguez-Leyva E, Ortega-Arenas LD, Huerta-de la Peña A (2011) Toxicidad de cuatro insecticidas sobre Tamarixia triozae (Burks) (Hymenoptera: Eulophidae) y su hospedero Bactericera cockerelli (Sulc) (Hemiptera: Triozidae). Acta Zoológica Mexicana 27: 509-526.

Liu TX, Zhang YM, Peng LN, Rojas P, Trumble JT (2012) Risk assessment of selected insecticides on Tamarixia triozae (Hymenoptera: Eulophidae), a parasitoid of Bactericera cockerelli (Hemiptera: Trizoidae). Journal of Economic Entomology 105: 490-496.

Mahdi SHA, Rahman MK (2008) Insecticidal effect of some spices on Callosobruchus maculatus (Fabricius) in black gram seeds. University Journal of Zoology, Rajshahi University 27: 47-50.

McCullagh P, Nelder JA (1989) Generalized linear model. Chapman \& Hall. London, UK. 511p.

McDougall CA (1986) Response of Diadegma terebrans (Gravenhorst) (Hymenoptera: Ichneumonidae) to berberine and alpha-terthienyl in the diet of its host, Ostrinia nubilalis (Hubner) (Lepidoptera: Pyralidae). University of Ottawa. Otawa, Canada. 145p. https://www.ruor.uottawa.ca/bitstream/10393/5163/1/ML33291.PDF. Fecha de consulta 15 de marzo de 2017.

Montes-Molina JA, Luna-Guido ML, Espinoza-Paz N, Govaerts B, Gutierrez-Miceli FA, Dendooven L (2008) Are extracts of neem (Azadirachta indica A. Juss. (L.)) and Gliricidia sepium Jacquin an alternative to control pests on maize Zea mays L.? Crop Protection 27: 763-774.

Morales-Ramos JA, Rojas MG (2003) Natural enemies and pest control: an integrated pest management concept. In: Koul O, Dhaliwal GS (Eds.). Predators and Parasitoids. Taylor \& Francis Group. London. pp. 17-39.

Ojimelukwe PC, Adler C (2002) Potentials of cinnamaldehyde and methylchavicol as grain protectants against four insect pests of stored products. IOBC WPRS Bulletin 25: 147-152.

Philogène BJR, Arnason JT, Berg CW, Duval F, Champagne D, Taylor RG, et al. (1985) Synthesis and evaluation of the naturally occurring phototoxin Alpha-terthienyl as a control agent for larvae of Aedes intrudens, Aedes atropalpus (Diptera: Culicidae) and Simulium verecundum (Diptera: Simuliidae). Journal of Economic Entomology 78: 121-126.

Pluke RW, Qureshi JA, Stansly PA (2008) Citrus flushing patterns, Diaphorina citri (Hemiptera: Psyllidae) populations and parasitism by Tamarixia radiata (Hymenoptera: Eulophidae) in Puerto Rico. Florida Entomologist 91: 36-42.

Qureshi JA, Rogers ME, Hall DG, Stansly PA (2009) Incidence of invasive Diaphorina citri (Hemiptera: Psyllidae) and its introduced parasitoid Tamarixia radiata (Hymenoptera: Eulophidae) in Florida citrus. Journal of Economic Entomology 102: 247-256.

Sidi MB, Islam MT, Ibrahim Y, Omar D (2013) Effect of azadirachtin and rotenone on Trichogramma papilionis (Hymenoptera: Trichogrammatidae). Journal of Food, Agriculture and Environment 11: 1509-1513.

Simmonds MSJ, Manlove JD, Blaney WM, Khambay BPS (2002) Effects of selected botanical insecticides on the behaviour and mortality of the glasshouse whitefly Trialeurodes vaporariorum and the parasitoid Encarsia formosa. Entomologia Experimentalis et Applicatta 102: 39-47

Tang YQ, Weathersbee III AA, Mayer RT (2002) Effect of neem seed extract on the brown citrus aphid (Homoptera: Aphididae) and its parasitoid Lysiphlebus testaceipes (Hymenoptera: Aphidiidae). Environmental Entomology 31: 172-176. 
Wang X, Xu H, Liu X, Zhao S (2000) The analysis of alpha-terthienyl in compositae plants and their bioactivity against mosquito larvae. Journal of South China Agricultural University 22: 26-28.

Wiesbrook ML (2004) Natural indeed: are natural insecticides safer and better than conventional insecticides. Illinois Pesticide Review 17: 1-8.

Xu YY, Liu TX, Leibee GL, Jones WA (2004) Effects of selected insecticides on Diadegma insulare (Hymenoptera: Ichneumonidae), a parasitoid of Plutella xylostella (Lepidoptera: Plutellidae). Biocontrol Science and Technology 14: 713-723. 